\title{
Interrupción legal del embarazo en la Ciudad de México: el papel de la educación
}

\section{Resumen}

Este trabajo estudia la asociación que existe entre el nivel educativo y la interrupción legal del embarazo mediante la implementación de una serie de modelos del tipo logit. Utilizando una muestra de más de 70,000 mujeres de entre 11 y 53 años de edad para el periodo comprendido entre enero 2016 y mayo 2020, se encuentra que las mujeres más educadas son más propensas a abortar por convicción y no por necesidad y/o presión social. Además, se muestra que existen otros factores relacionados al aborto inducido, tales como el acceso a los servicios de salud, la remuneración en el trabajo, así como el uso de anticonceptivos, la edad de inicio de la vida sexual, el número de hijos, el número de abortos previos y la religión. El análisis confirma varios de los resultados obtenidos previamente en la literatura empírica y documental y aporta al debate sobre la despenalización sobre el aborto en México.

\section{Introducción}

El aborto inducido es una práctica que se ha desarrollado desde mucho antes de que existieran los registros históricos (Sagot-Rodríguez \& Carcedo Cabañas, 2002). El tema ha sido objeto de intensos debates entre la comunidad científica y sociedad en general. En estos se identifican dos posturas: la liberal y la conservadora (Taracena, 2005). La primera se refiere a la adjudicación de un derecho absoluto de las mujeres para decidir sobre su propio cuerpo y la 
segunda a la adjudicación de un derecho absoluto para la vida del cigoto. Estas posturas se asocian con diferentes factores, entre los cuales se encuentran los de carácter ético, religioso, político, jurídico, entre otros. Entre la comunidad médica también se han detectado otros factores como el género, la edad o incluso la especialidad (González, 1995). Sin embargo, lejos de toda esta discusión, es innegable que el aborto es una práctica que seguirán desarrollando las mujeres del todo el mundo, a pesar de estar tan estigmatizada.

Se estima que en el periodo 2015-2019 hubieron, en promedio, alrededor de 73 millones de abortos a nivel mundial, lo que corresponde a una tasa de 39 casos por cada 1,000 mujeres de entre 15 y 44 años. Esta tasa no solo ha variado con el tiempo, sino que también depende de la región y del nivel de ingreso. Por ejemplo, en el oeste de Asia la tasa de abortos era de 57 casos por cada 1,000 mujeres para el periodo 2015-2019, mientras que en Norte América era de solo 12 casos por cada 1,000 mujeres (Bearak et al., 2020). La tasa de abortos en México se estimaba en 38 casos por cada 1,000 mujeres de entre 15 y 44 años para el año 2009 (Juarez et al., 2013); la tasa más elevada se observa entre las mujeres de 20 a 24 años, seguida por el grupo de mujeres de entre 15 y 19 años. Además, la entidad federativa con la tasa más alta de abortos fue el Distrito Federal (hoy Ciudad de México) y la entidad con la tasa más baja fue Nuevo León (Juarez et al., 2013).

A pesar de altas tasas que se observan hoy en día, el aborto no es legal en todos los países y/o regiones del mundo. En particular, se estima que alrededor del 5\% de las mujeres en edad reproductiva viven en países que prohiben el aborto completamente, mientras que el $37 \%$ viven en países en los que el aborto está despenalizado. ${ }^{1}$ México pertenece al grupo de países que permiten el aborto en casos necesarios, aunque las leyes específicas dependen de cada Entidad Federativa. Algunos de los causales de aborto en México son la violación, el peligro de muerte, los daños irreversibles para la madre y las malformaciones graves de

\footnotetext{
${ }^{1}$ Center for Reproductive Rights. The World's Abortion Laws. Disponible en https://maps . reproductiverights.org/worldabortionlaws. Consultado el 11 de agosto de 2021.
} 
feto. Solo la Ciudad de México, Oaxaca y muy recientemente Hidalgo permiten a las mujeres abortar hasta las 12 semanas de gestación de manera legal sin importar la causa; esto es, permiten que una mujer aborte por convicción y no necesariamente por necesidad.

En la literatura se han identificado varios factores socioeconómicos asociados al aborto en condiciones de legalidad. Entre estos se encuentran el género, la edad, el lugar de nacimiento, la posición social, entre otros. Este trabajo se interesa principalmente en la educación como variable asociada a la decisión de abortar. En general, se espera que las mujeres menos educadas tengan mayores tasas de aborto, pero que sean las mujeres más educadas las más propensas a abortar por convicción y no por necesidad y/o presión social. Para probar esta hipótesis, se utilizan las bases de datos de la Ciudad de México, la cual lleva un registro de las solicitudes de aborto, culminado o no, en las Clínicas de Salud Sexual y Reproductivas de la Secretaría de Salud de la entidad, e implementamos una serie de modelos del tipo logit.

Aunque no se han encontrado estudios previos que utilicen la metodología y la base de datos aquí presentados, sí existen diversos trabajos relacionados. Los principales son (Eskild et al., 2007) y (González-Rábago et al., 2017). El primero usa una muestra de 94,428 mujeres Noruegas y 5,390 mujeres Pakistaníes para analizar el efecto de la educación sobre la decisión de abortar utilizando la edad, el número de embarazos previos, el estado civil y el lugar de residencia como variables de control. En el caso de las mujeres Pakistaníes no se encontró asociación entre la educación y el aborto inducido, pero para las mujeres Noruegas se encontró que los niveles más bajos de educación se asocian con una mayor frecuencia de aborto. Estos resultados se confirman en (González-Rábago et al., 2017), en el que se usa una muestra de 11,946 mujeres Españolas. En (González-Rábago et al., 2017), además de encontrar una asociación inversa entre la frecuencia de aborto y los niveles educativos, también se encuentra que las mujeres menos educadas tienen más probabilidades de abortar en más de una ocasión. En la literatura también pueden encontrarse otros estudios más generales del aborto inducido. 
Por ejemplo, (Figueroa-Lara et al., 2012) realiza un estudio de casos y controles para mostrar que, además de los años de escolaridad, otras variables asociadas al aborto inducido son la ocupación y el número de embarazos previos. También se ha reconocido a la religión como un factor preponderante de la decisión de interrumpir un embarazo. Esto se relaciona a la presión que ejerce la religión desde un punto de vista personal y político (Lamas, 2009). Otros posibles factores encontrados en la literatura son la edad, el número de hijos, el consumo de alcohol, el maltrato, el estado civil y el deseo de suicidio (González Poveda \& Rodríguez Cabra, 2015), así como factores asociados a la economía, a la pareja y a los proyectos de vida (Prada et al., 2011). En el caso de adolescentes, también se han encontrado otras variables relacionadas con la decisión de abortar, tales como el inicio precoz de la sexualidad, la falta de información sobre el uso de métodos anticonceptivos, la promiscuidad, la presencia de familiares adolescentes con embarazos, los hogares monoparentales, así como la presión ejercida por la familia (Doblado Donis et al., 2010).

La base de datos que se utiliza en este trabajo permite asociar el nivel educativo a la decisión de abortar de forma voluntaria e incluir gran parte de las variables mencionadas líneas arriba como variables de control. Los resultados confirman la hipótesis planteada. Esto es, las mujeres menos educadas tienen mayores tasas de aborto, pero son las mujeres más educadas las más propensas a abortar por convicción. Además, se muestra que las mujeres en condiciones de vida menos favorables abortan principalmente por necesidad y/o por situaciones que ponen en riesgo su vida o la del feto. Asimismo, se encuentra que la edad, el uso de anticonceptivos, la edad de inicio de la vida sexual, el número de hijos, el número de abortos previos, así como la religión, son factores relacionados con la decisión de abortar.

El resto del estudio se distribuye como sigue. Seguido de esta introducción, se describen los datos y la metodología utilizados en la Sección . Luego, en la Sección se discuten los resultados. Finalmente, se concluye en la Sección . 


\section{Datos y metodología}

En este trabajo se utiliza el registro de las solicitudes de aborto, culminado o no, en las Clínicas de Salud Sexual y Reproductivas de la Secretaría de Salud de la Ciudad de México. La base de datos se compone de información sobre 70,390 mujeres de entre 11 y 53 años de edad para el periodo comprendido entre enero 2016 y mayo 2020. Al tratarse de un registro y no de una encuesta, esta muestra no puede considerarse representativa de todas las mujeres que abortan en México, pero, por su propia naturaleza, sí puede considerarse representativa de todas las mujeres que abortan en condiciones de legalidad. Esta información es pública y puede consultarse de forma gratuita en el portal de datos abiertos del Gobierno de la Ciudad de México. ${ }^{2}$

Se consideran las siguientes variables:

- Estado Civil

- Edad

- Acceso a servicios de salud

- Ocupación

- Religión

- Edad de inicio de vida sexual activa

- Número de hijos

- Número de abortos

- Número de interrupciones legales del embarazo previas

- Consejería

- Uso de anticonceptivo previo al embarazo

- Nivel educativo

\footnotetext{
${ }^{2}$ Ver: https://datos.cdmx.gob.mx/dataset/interrupcion-legal-del-embarazo. Consultado el 11 de agosto de 2021.
} 
- Motivos para desea la interrupción del embarazo

Esta última variable representa los diferentes motivos reportados por las mujeres para abortar. Entre estos se incluyen los causales de aborto legal en todo el país, tales como malformación del feto, riesgo de vida para la madre y violación, así como condiciones socioeconómicas. Cuando la mujer no reporta ninguna razón particular para abortar, la variable de motivos se codifica simplemente como interrupción voluntaria. Se puede pensar en una interrupción voluntaria como una interrupción por convicción. Dado que es precisamente esta posibilidad de abortar por convicción lo que hace diferente a la Ciudad de México, Oaxaca e Hidalgo del resto del país, para el realizar el análisis se ha construido una variable binaria que toma el valor de 1 en el caso de interrupción voluntaria y 0 de otra forma. Esta variable binaria es la variable dependiente.

Algunas de las variables arriba mencionadas también fueron reconfiguradas en la forma de variables binarias, de tal forma que tengan una mejor interpretación. En particular, se ha construido una variable binaria que toma el valor de 1 si la mujer reportó estar casada y 0 si no. La variable de acceso a servicios de salud toma el valor de 1 si se reportó acceso a cualquier de los servicios de salud disponibles en México y 0 si no. Entre los servicios de salud se encuentran el Instituto Mexicano del Seguro Social, el Seguro Popular, el ISSSTE, y otros. También se tiene una variable binaria que toma el valor de 1 si la mujer tiene una ocupación remunerada y otra binaria que toma el valor de 1 si la mujer profesa la religión católica. Otras variables binarias son la de consejería, que toma el valor de 1 si la mujer recibió atención del centro de consejería de las Clínicas de Salud Sexual y Reproductivas y la de anticonceptivos, que toma el valor de 1 si la mujer reportó haber usado algún método anticonceptivo.

Con respecto al nivel educativo, esta, de origen, reporta el último nivel escolar acreditado, el cual va desde ningún nivel de escolaridad hasta doctorado. Para realizar el ejercicio, se 
han construido un conjunto de variables binarias educativas por nivel: 1) Sin educación, la cual toma el valor de 1 si la mujer no acreditó ningún nivel de escolaridad y 0 de otra forma; 2) Básica, la cual toma el valor de 1 si la mujer acreditó primaria o secundaria y 0 de otra forma; 3) Media superior, que toma el valor de 1 si la mujer acreditó estudios de nivel medio superior y 0 de otra forma; 4) Superior, que toma el valor de 1 si la mujer acreditó estudios de nivel superior y 0 de otra forma; 5) Posgrado, que toma el valor de 1 si la mujer acreditó estudios de maestría o doctorado y 0 de otra forma. Adicionalmente, se ha creado una variable binaria que toma el valor de 1 si la mujer acreditó cualquier nivel educativo por encima del medio superior, incluyendo este, y 0 en caso de haber cursado solo educación básica.

\section{Estadística descriptiva}

La Tabla 1 describe la variable sobre interrupción legal del embarazo (ILE), la variable dependiente, para la muestra considerada. Del total de personas con información disponible, 98.54\% no dieron una razón particular para abortar. Estos casos se consideran de aborto por convicción. El restante 1.46\% dio una razón relacionada con su salud o la del feto, violación, o cualquiera otra de las razones que permiten que una mujer aborte en todo el país.

\begin{tabular}{llr} 
& Cuadro 1: Variable dependiente: Interrupción legal del embarazo (ILE) \\
\hline & & \#Total \\
\hline ILE & Interrupción por convicción & $98.54 \%$ \\
& Otro motivo de interrupción & $1.46 \%$ \\
& Total de casos & 69,722 \\
\hline
\end{tabular}

Fuente: Elaboración propia con datos del Gobierno de la Ciudad de México

La Tabla 2 describe las variables educativas que han sido construidas para realizar el análisis. Se puede destacar, por ejemplo, que solo el $1 \%$ de las mujeres en busca de ILE no acreditaron ningún nivel educativo, mientras que el 35\% cuenta con educación básica (primaria o 
secundaria), 45\% cuenta con educación media superior, $18 \%$ cuenta con educación superior y tan solo alrededor del $0.5 \%$ cuenta con estudios de posgrado, ya sea maestría y/o doctorado.

Cuadro 2: Variables binarias educativas

\begin{tabular}{rlr}
\hline & & \#Total \\
\hline Educación & Con Educación & $99.01 \%$ \\
& Sin educación & $0.99 \%$ \\
& Total de casos & 69,935 \\
Educación básica & Otro & $65.05 \%$ \\
& Con educación básica & $34.95 \%$ \\
& Total de casos & 69,935 \\
Educación media superior & Otro & $54.77 \%$ \\
& Con educación media superior & $45.23 \%$ \\
& Total de casos & 69,935 \\
Educación superior & Otro & $81.63 \%$ \\
& Con educación superior & $18.37 \%$ \\
& Total de casos & 69,935 \\
Posgrado & Otro & $99.54 \%$ \\
& Con posgrado & $0.46 \%$ \\
& Total de casos & 69,935 \\
Mayor a media superior & Educación básica & $35.94 \%$ \\
& Educación media superior y superior & $64.06 \%$ \\
& Total de casos & 69,935 \\
\hline
\end{tabular}

Fuente: Elaboración propia con datos del Gobierno de la Ciudad de México

La Figura 1 hace un resumen gráfico de las principales estadísticas de las variables de control que serán consideradas en el modelo. En la parte superior se muestran las variables discretas y en la inferior las variables continuas. Un resumen numérico se muestra en la Tabla 3. 


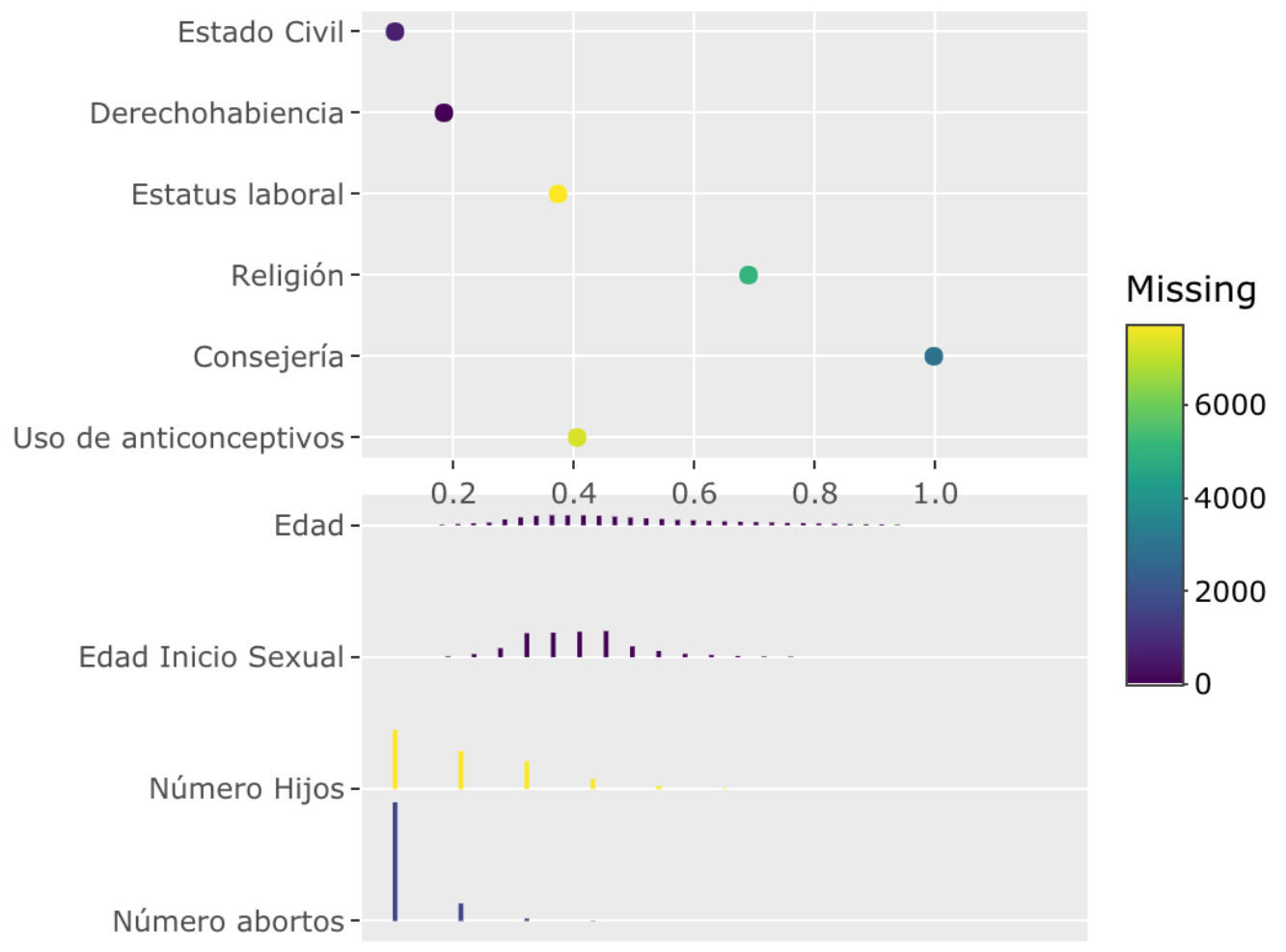

Figura 1: Estadística descriptiva: variables de control

La Figura 1 y la Tabla 3 revelan, por ejemplo, que:

- Si bien la muestra contiene mujeres de todas las edades buscando el servicio de ILE, el número servicios se concentra en mujeres de entre 18 y 32 años. De acuerdo a la Tabla 3, la media de edad es 25.7 años.

- La mayor parte de las mujeres inician su vida sexual entre los 14 y 20 años, siendo la media de 17 años.

- Una gran cantidad de mujeres que buscan abortar no tienen otros hijos o tienen muy pocos. Una persona reportó tener 10 hijos al momento de solicitar el servicio de ILE.

- La mayor parte de las mujeres que solicitan el servicio de ILE no reportaron otros abortos. Sin embargo, 3 personas reportaron 10 abortos previos. 
- Alrededor del $10 \%$ de las mujeres están casadas y $18.5 \%$ tienen acceso a algún servicio formal de salud.

- Alrededor del $40 \%$ de las mujeres reportan haber usado algún método anticonceptivo. Es de destacar que en la base de datos existen mujeres que declararon haber usado más de un método anticonceptivo simultáneo, lo cual deja en claro su intención de no tener hijos.

- Alrededor del 37\% de las mujeres tienen un empleo remunerado. El resto de las mujeres o no trabaja, o estudia, o trabaja sin remuneración formal.

- Alrededor del $70 \%$ de las mujeres han declarado profesar la religión católica y prácticamente todas recibieron consejos sobre el proceso de aborto voluntario.

Cuadro 3: Estadística descriptiva: variables de control

\begin{tabular}{rlr}
\hline & & \#Total \\
\hline Edad & Media & 25.72 \\
& Desviación Estándar & 6.27 \\
Edad inicio sexual & Media & 17.00 \\
& Desviación Estándar & 2.31 \\
Número hijos & Media & 1.02 \\
& Desviación Estándar & 1.13 \\
Número abortos & Media & 0.18 \\
& Desviación Estándar & 0.47 \\
Estado Civil & No casada & $89.68 \%$ \\
& Casada & $10.32 \%$ \\
Derechohabiencia & Sin acceso a servicios de salud & $81.55 \%$ \\
& Acceso a servicios salud & $18.45 \%$ \\
Estatus laboral & Trabajo no remunerado & $62.63 \%$ \\
& Trabajo remunerado & $37.37 \%$ \\
Religión & No católica & $31.01 \%$ \\
& Católica & $68.99 \%$ \\
Consejería & Sin consejería & $0.26 \%$ \\
& Consejería & $99.74 \%$ \\
Uso anticonceptivos & Sin uso de anticonseptivos & $59.46 \%$ \\
& Uso de anticonceptivos & $40.54 \%$ \\
\hline
\end{tabular}

Fuente: Elaboración propia con datos del Gobierno de la Ciudad de México 


\section{Metodología}

Los modelos de elección binaria permiten responder a la siguiente pregunta: ¿cuál es la probabilidad de que una mujer busque una $I L E$ condicionando a los diferentes niveles de las variables de control?

Sea $Y_{i}$ la variable dependiente. En el modelo que aquí se presenta, $Y_{i}=1$ indica que la $i$ ésima persona, $i=1, \ldots, n$, intenta abortar por convicción y $Y_{i}=0$ de otra forma. Sea $X$ el conjunto de variables de control o predicción en el modelo; esta es una matriz de dimensión $n \times k$. Para calcular la probabilidad de aborto legal, $P\left(Y_{i}=1 \mid X\right)$, es necesario restringir esta al intervalo $[0,1]$. Esto se logra mediante la siguiente especificación:

$$
P\left(Y_{i}=1 \mid X\right)=F(X \beta)
$$

donde $\beta$ es el vector de dimensión $k \times 1$ de coeficientes a estimar y $F(\cdot)$ cumple con las propiedades:

$$
\begin{array}{r}
F(-\infty) a m p ;=0 \\
F(+\infty) a m p ;=1 \\
f(X)=\frac{\partial F(X)}{\partial X} a m p ;>0
\end{array}
$$

En otras palabras, $F(\cdot)$ es una función diferenciable monótona creciente con dominio real y rango $[0,1]$.

En el caso de este estudio, se supondrá que $F(\cdot)$ es la función de distribución logística. Si $L$ es una variable aleatoria que proviene de esta distribución, entonces:

$$
P\left(Y_{i}=1 \mid X\right)=P(L \leq X \beta)=\Lambda(X \beta)=\frac{\exp (X \beta)}{1+\exp (X \beta)}
$$


Naturalmente:

$$
P\left(Y_{i}=0 \mid X\right)=1-P(L \leq X \beta)=\frac{1}{1+\exp (X \beta)}
$$

Estas probabilidades pueden estimarse mediante el método de máxima verosimilitud.

Una de las ventajas de usar la función de densidad logística es que es posible expresar los resultados en términos del cociente de probabilidades, también conocido como razón de momios, de la siguiente forma:

$$
\frac{P\left(Y_{i}=1 \mid X\right)}{P\left(Y_{i}=0 \mid X\right)}=\exp (X \beta) \geq 0
$$

Este cociente indica cuál es la probabilidad de que $Y_{i}=1 \mid X$ con respecto a la probabilidad que $Y_{i}=0 \mid X$. Este cociente es mayor o igual que 0, pero el punto de referencia es 1. Por ejemplo, si este cociente es mayor a 1 tenemos que la probabilidad de elegir un aborto por convicción es mayor en relación a la probabilidad de elegir otra opción. Lo contrario es verdad si el cociente es menor que 1.

Contrario a la costumbre en Economía de presentar los resultados en términos de los coeficientes marginales, aquí se prefiere presentarlos en términos del cociente de probabilidades. Esto es porque, al menos para el caso particular de este estudio, los resultados se leen e interpretan de una forma más intuitiva. Este análisis se realiza en la siguiente sección.

\section{Resultados}

La metodología anterior se usa para construir un modelo en el que la variable dependiente, ILE, la cual es una variable binaria que toma el valor de 1 si la mujer aborta sin motivo particular (por convicción) y 0 de otra forma, se relaciona con el resto de las variables des- 
critas en la Sección, principalmente con las variables educativas. Los resultados se muestran en la Tabla 4.

En el Modelo 1 de la Tabla 4 se han incluido las variables binarias educativas por nivel. En este modelo la referencia son las mujeres que no han acreditado ningún nivel educativo. En el Modelo 2, en lugar de utilizar las variables educativas por nivel, se ha incluido la variable binaria que indica si una mujer acreditó el nivel medio superior o más. El Modelo 3 es una extensión del Modelo 2 en el cual se ha agregado la interacción entre el número de hijos y el número de abortos. En la Tabla 4 se presenta el cociente de probabilidades para las variables de control; los errores estándar se muestran entre paréntesis.

Como muestra la Tabla 4, las variables educativas por nivel no son estadísticamente significativas, lo cual implica que el nivel educativo no está asociado con la elección de abortar por convicción. Esto constituye, en principio, una contradicción a la hipótesis planteada al inicio de este trabajo. Sin embargo, cuando se considera a la variable binaria que indica la acreditación del nivel medio superior o más, se observa que esta sí estadísticamente significativa. Esto puede deberse a que es precisamente a partir del nivel medio superior cuando las mujeres comienzan a tener más acceso a la educación sexual y a tomar más conciencia de las posibles consecuencias de un embarazo. Asimismo, las mujeres más educadas dejan, potencialmente, de ser víctimas de la presión social que conlleva un potencial aborto luego de un embarazo no planeado.

La Tabla 4 también revela otras variables que no son estadísticamente significativas. Este es el caso de las variables que indican el estado civil y la recepción del servicio de consejería sobre el proceso de ILE. El primero de estos resultados podría indicar que la decisión de aborto corresponde exclusivamente a la mujer. El segundo podría indicar que, una vez que la mujer toma la decisión de abortar, cualquier consejo sobre el proceso médico o intento de persuasión se hace irrelevante. 
Cuadro 4: Resultados

\begin{tabular}{|c|c|c|c|}
\hline & \multicolumn{3}{|c|}{ Variable dependiente: } \\
\hline & \multicolumn{3}{|c|}{ Interrupción legal del embarazo } \\
\hline & Modelo 1 & Modelo 2 & Modelo 3 \\
\hline & (1) & $(2)$ & (3) \\
\hline Estado Civil & $\begin{array}{c}0.895 \\
(0.156)\end{array}$ & $\begin{array}{c}0.896 \\
(0.156)\end{array}$ & $\begin{array}{c}0.896 \\
(0.155)\end{array}$ \\
\hline Edad & $\begin{array}{c}1.060 \\
(0.011)^{* * *}\end{array}$ & $\begin{array}{c}1.058 \\
(0.011)^{* * *}\end{array}$ & $\begin{array}{c}1.061 \\
(0.011)^{* * *}\end{array}$ \\
\hline Derechohabiencia & $\begin{array}{c}7.566 \\
(0.263)^{* * *}\end{array}$ & $\begin{array}{c}7.566 \\
(0.263)^{* * *}\end{array}$ & $\begin{array}{c}7.620 \\
(0.263)^{* * *}\end{array}$ \\
\hline Educación basica & $\begin{array}{c}0.374 \\
(0.715)\end{array}$ & & \\
\hline Educación media superior & $\begin{array}{c}0.498 \\
(0.715)\end{array}$ & & \\
\hline Educación superior & $\begin{array}{c}0.424 \\
(0.720)\end{array}$ & & \\
\hline Posgrado & $\begin{array}{c}125,318.000 \\
(259.909)\end{array}$ & & \\
\hline Media superior o más & & $\begin{array}{c}1.256 \\
(0.105)^{* *}\end{array}$ & $\begin{array}{c}1.251 \\
(0.105)^{* *}\end{array}$ \\
\hline Estatus laboral & $\begin{array}{c}1.252 \\
(0.107)^{* *}\end{array}$ & $\begin{array}{c}1.251 \\
(0.107)^{* *}\end{array}$ & $\begin{array}{c}1.257 \\
(0.107)^{* *}\end{array}$ \\
\hline Religion & $\begin{array}{c}0.671 \\
(0.115)^{* * *}\end{array}$ & $\begin{array}{c}0.669 \\
(0.115)^{* * *}\end{array}$ & $\begin{array}{c}0.669 \\
(0.115)^{* * *}\end{array}$ \\
\hline Inicio vida sexual & $\begin{array}{c}0.850 \\
(0.019)^{* * *}\end{array}$ & $\begin{array}{c}0.850 \\
(0.019)^{* * *}\end{array}$ & $\begin{array}{c}0.848 \\
(0.019)^{* * *}\end{array}$ \\
\hline Número hijos & $\begin{array}{c}0.827 \\
(0.054)^{* * *}\end{array}$ & $\begin{array}{c}0.833 \\
(0.053)^{* * *}\end{array}$ & $\begin{array}{c}0.788 \\
(0.056)^{* * *}\end{array}$ \\
\hline Número abortos & $\begin{array}{c}0.770 \\
(0.081)^{* * *}\end{array}$ & $\begin{array}{c}0.770 \\
(0.081)^{* * *}\end{array}$ & $\begin{array}{c}0.564 \\
(0.125)^{* * *}\end{array}$ \\
\hline Consejería & $\begin{array}{c}1.467 \\
(1.014)\end{array}$ & $\begin{array}{c}1.493 \\
(1.014)\end{array}$ & $\begin{array}{c}1.635 \\
(1.013)\end{array}$ \\
\hline
\end{tabular}


De las variables que son estadísticamente significativas, tomemos primero a la derechohabiencia, y al estatus laboral. Estas fueron definidas como variables binarias, de tal forma que un cociente de probabilidades mayor que 1 describe una relación positiva. Por ejemplo, las mujeres con acceso a los servicios formales de salud tienen 7.5 más de probabilidades de abortar sin un motivo particular que las mujeres sin acceso a estos servicios. De igual forma, las mujeres con trabajos remunerados tienen 1.25 veces más de probabilidades de elegir interrupciones del embarazo por convicción. Estos resultados, en conjunto con los resultados sobre las variables educativas, indican que las mujeres en situación de desventaja, es decir, sin información o menos educadas y en condiciones de vida menos favorables, abortan solo por necesidad y/o por situaciones que ponen en riesgo su vida o la del feto.

En el caso de la edad de las mujeres que asisten a las Clínicas de Salud Sexual y Reproductivas, la Tabla 4 indica que con cada año adicional la probabilidad de abortar por convicción se incrementa por 1.06. Este es un indicativo de la importancia que tiene la madurez de la persona ante una situación de posible aborto.

En la Tabla 4 también se encuentran variables estadísticamente significativas con cocientes de probabilidades inferiores a 1. Este es el caso de las variables binarias que indican religión y el uso de métodos anticonceptivos. En particular, tenemos que las mujeres que declararon profesar una religión diferente a la católica tienen aproximadamente 1.5 (1/0.66) veces más de probabilidades de abortar por convicción. Este resultado no es de extrañar y va de la mano con la creencia católica de que la vida humana es sagrada desde la fecundación del óvulo por el espermatozoide, por lo que el ser humano no puede intervenir para interrumpirla, pues ello significa ir contra la voluntad de Dios (Taracena, 2005). Por otro lado, las mujeres que usaron métodos anticonceptivos tienen aproximadamente 4.5 (1/0.225) veces más de probabilidades de abortar por convicción en relación a las que no usaron métodos anticonceptivos. Esto se debe a la relación evidente que existe entre el número de embarazos no deseados y el aborto 
inducido (Juarez et al., 2013).

Finalmente, los cocientes de probabilidad inferiores a uno para la edad de inicio de vida sexual activa, el número de hijos y el número de abortos indican que las mujeres que iniciaron más jóvenes su vida sexual, que tienen menos hijos y que han sufrido de menos abortos tienen más probabilidades de abortar por convicción. Adicionalmente, el Modelo 3 en la Tabla 4 indica que también la interacción entre el número de hijos y el número de abortos es estadísticamente significativa, pero tiene un cociente de probabilidades superior a 1. Esto implica que, por ejemplo, el efecto marginal del número de abortos sobre la probabilidad de abortar por convicción no es constante, más bien depende del número de hijos que tenga la mujer. En particular, entre más hijos, menos el efecto decreciente del número de abortos.

\section{Discusión y conclusiones}

En este trabajo se estudia la asociación que existe entre el nivel educativo y la interrupción legal del embarazo mediante la implementación de una serie de modelos del tipo logit. A diferencia de la mayor parte de la literatura existente, en la cual se estudia al aborto inducido mediante muestras de no más de 500 observaciones, aquí se ha utilizado una muestra de más de 70,000 mujeres de entre 11 y 53 años de edad para el periodo comprendido entre enero 2016 y mayo 2020. Esta información proviene de las bases de datos abiertos de la Ciudad de México.

A diferencia de los resultados encontrados por estudios similares a este, los cuales indican que las mujeres menos educadas tienen las tasas más altas de aborto (Eskild et al., 2007; González-Rábago et al., 2017), los resultados aquí obtenidos indican que son las mujeres más educadas las más propensas a abortar por convicción y no por necesidad y/o presión social. Específicamente, se ha encontrado que existe una diferencia estadísticamente significativa 
entre la probabilidad que tienen las mujeres de abortar por convicción cuando estas tienen educación media superior o más en relación a aquellas que solo han acreditado el nivel básico. Ambos resultados no son contradictorios, sino complementarios. El primero de ellos se encuentra cuando la muestra incluye mujeres que están decidiendo si abortar o no; el segundo se obtiene cuando la base de datos está conformada, como ha sido el caso aquí, por mujeres que ya han decidido abortar y se desean analizar por las razones por las cuales han tomado esta decisión.

Los resultados obtenidos pueden explicarse desde, al menos, dos puntos de vista interrelacionados. Primero, si bien en México la educación sexual comienza desde la educación básica, existe evidencia de que esta es usualmente incompleta, especialmente a nivel primaria (Rojas et al., 2017). Es a partir de la educación media superior que las mujeres tienen acceso a toda la información sobre salud sexual y toman más conciencia sobre las consecuencias de un embarazo no deseado y, por supuesto, de un posible aborto.

El papel de la educación sexual se refuerza si se considera la relación evidente que existe entre el número de embarazos no deseados y el aborto inducido (Juarez et al., 2013). En este estudio, un embarazo no deseado puede entenderse como una persona que decide solicitar el servicio de aborto inducido, pero que reportó haber usado algún método anticonceptivo. Los resultados obtenidos indican una asociación positiva entre estas dos variables, lo cual deja en claro que una educación sexual integral en cualquier etapa de vida, especialmente en edades tempranas, es un factor crucial para la prevención de embarazos no deseados y, a su vez, para la reducción de las cifras de interrupciones voluntarias del embarazo.

Segundo, las mujeres que abortan luego de un embarazo no planeado, sobre todo las de menor edad y en condiciones de pobreza, referida esta no solo a la escasez de recursos económicos sino también a recursos sociales como la educación, relacionales, de información, entre otros (Erviti, 2005), tienden a ser etiquetadas como promiscuas, pecaminosas, 
egoístas, irresponsables o asesinas, lo cual resulta en miedo a la discriminación y la exclusión social (Kumar et al., 2009). De acuerdo a los resultados del presente estudio, este proceso de estigmatización y condena social puede atenuarse, al menos parcialmente, a través de la educación.

También puede destacarse el papel que juega la Iglesia Católica en la decisión de abortar. En la literatura se ha identificado a esta como uno de los principales actores que obstaculizan la ampliación del acceso al aborto legal y como uno de los principales movilizadores de laicos en defensa de la vida (Irrazábal, 2015). Los resultados obtenidos confirman esta observación. En particular, parece que la religión católica intensifica el proceso de estigmatización y culpa que conlleva un aborto, razón por la cual el modelo que se ha presentado identifica al catolicismo como un freno al aborto por convicción.

Del análisis también pueden obtenerse otros resultados relevantes. Primero se tiene el efecto de la madurez emocional. Existen estudios que demuestran que el embarazo en la adolescencia contribuye a la perpetuacion del ciclo de la pobreza, esto causado por la falta de oportunidades y aplanamiento de las perspectivas futuras de vida de la adolescente embarazada, colocando a las jovenes en un riesgo mas alto para alcanzar sus logros educativos, ocupacionales y socioeconomicos (García Odio \& González Suárez, 2018). Naturalmente, las mujeres más jóvenes tampoco están preparadas para tomar la decisión de abortar por convicción. En este estudio, el efecto de la madurez emocional está capturado por la variable edad. En particular, entre más joven es la mujer, menos la probabilidad de abortar por convicción. Los resultados obtenidos también confirman algunas de las tendencias analizadas por la literatura a lo largo de los años. Por ejemplo, se ha comprobado el deseo de las mujeres por formar familias cada vez más pequeñas (Juarez et al., 2013). Esto se refleja en una probabilidad de abortar por convicción mayor entre menor sea el número de hijos que ya se tienen. 
El presente análisis tiene algunas limitaciones. Primero, la naturaleza de los datos con los que se cuenta permite encontrar una asociación entre la educación y el aborto por convicción, pero no permite establecer un patrón de causalidad. Segundo, podría existir un problema de causalidad inversa si el acceso al aborto legal afecta al nivel educativo. Por ejemplo, (Rodgers et al., 2021) muestra que el acceso al aborto legal no solo podría disminuir los costos de atención médica en caso de complicaciones, sino que además podría resultar en un mejor desempeño escolar, en mejores tasas de participación de las mujeres en el mercado laboral y en menores tasas criminales. Tercero, debido a que la base de datos utilizada es un registro de casos y no una encuesta, no se cuenta necesariamente con una muestra representativa de todas las mujeres que abortan en México, aunque sí puede considerarse como una muestra representativa de todas las mujeres que abortan en condiciones de legalidad. Cuarto, existen algunas otras variables que no están disponibles en el registro de las Clínicas de Salud Sexual y Reproductivas de la Secretaría de Salud de la Ciudad de México que podrían ayudar en la explicación de la decisión de abortar. Entre estas se encuentran las variables socioeconómicas de los solicitantes, tales como el quintil de ingreso, condición de marginalidad y/o indigenismo, así como variables que permitan caracterizar a los médicos tratantes. Quinto, el registro de las Clínicas de Salud Sexual y Reproductivas de la Secretaría de Salud de la Ciudad de México reporta el último nivel escolar acreditado de las mujeres solicitantes del servicio de aborto en lugar de los años de escolaridad. Los años de escolaridad podrían permitir que en el modelo se incluyan algunas interacciones que podrían ser útiles para explicar mejor la interrelación entre la educación y las variables de control. Finalmente, los resultados presentados deben tomarse con cautela, pues un análisis más general debería considerar a todas las mujeres que se enfrentan a la decisión de abortar. Esto, sin embargo, no es posible debido a que se desconoce el número exacto y las características de las mujeres que abortan clandestinamente.

A pesar de estas limitaciones, los resultados del presente estudio aportan al debate sobre la 
legalización del aborto en México y justifican en parte los esfuerzos realizados por el Gobierno Mexicano y otros organismos nacionales e internacionales para promover la educación integral de la sexualidad y su incorporación al sistema educativo desde los primeros niveles. Además, se ha mostrado que la educación en sí misma podría ser un factor que disminuya la carga emocional a la que se someten las mujeres que deciden abortar. Lo anterior tiene importantes implicaciones en la elaboración de políticas públicas, pues estas tendrían que ocuparse, primero, en proporcionar un nivel educativo lo suficientemente alto que le permita a la sociedad asimilar un sistema de salud en el que las mujeres pueda elegir libremente sobre el momento, las razones y las condiciones para llevar a cabo un aborto.

\section{Referencias}

Aborto inducido: ética y derechos. (2002). Medicina Legal De Costa Rica, 19.

El aborto a debate. Análisis de los argumentos de liberales y conservadores. (2005). Desacatos, 17.

[The physicians and abortion].. (1995). Salud Publica Mex, 37, 248-255.

Unintended pregnancy and abortion by income, region, and the legal status of abortion: estimates from a comprehensive model for 1990-2019. (2020). Lancet Glob Health, 8, e1152-e1161.

Embarazo no planeado y aborto inducido en Mexico: causas y consecuencias. (2013). Guttmacher Institute.

Childbearing or induced abortion: the impact of education and ethnic background. Population study of Norwegian and Pakistani women in Oslo, Norway.. (2007). Acta Obstet Gynecol Scand, 86, 298-303. 
The role of birthplace and educational attainment on induced abortion inequalities.. (2017). BMC Public Health, 17, 69.

[Factors associated with the seeking of legal induced abortion services in Mexico City in 2010].. (2012). Salud Publica Mex, 54, 401-409.

La despenalización del aborto en México - Nueva Sociedad. (2009). Nueva Sociedad, 220.

Aborto: las variables que están asociadas a tomar esta decisión en el caso colombiano. (2015).

Embarazo no deseado y aborto inducido en Colombia: causas y consecuencias. (2011). New York: Guttmacher Institute.

Aborto en la adolescencia un problema de salud. (2010). Revista Cubana De Obstetricia y Ginecología, 36(3).

Educación sexual integral: cobertura homogeneidad, integralidad y continuidad en escuelas de México. (2017). Salud PúBlica De MéXico, 59(1), 19. https://doi.org/10.21149/8411

El aborto entre mujeres pobres. (2005). Universidad Nacional Autónoma de México.

Conceptualising abortion stigma.. (2009). Cult Health Sex, 11, 625-639.

La religión en las decisiones sobre aborto no punible en la Argentina. (2015). Revista Estudos Feministas, 23(3), 735-759. https://doi.org/10.1590/0104-026x2015v23n3p735

Factores de riesgo asociados a embarazadas adolescentes. (2018). Revista De Ciencias Médicas Del Pinar Del Rio, 22(3).

The macroeconomics of abortion: A scoping review and analysis of the costs and outcomes.. (2021). PLoS One, 16, e0250692. 